\title{
Advances in Auger Electron Spectroscopy and its Applications in the Field of Nanotechnology
}

\author{
S. N. Raman, D. F. Paul, J. S. Hammond and D. G. \\ Watson Physical Electronics, Inc., 18725 Lake Drive East, Chanhassen Minnesota, 55317
}

The field of nanotechnology has expanded with advances in high resolution imaging. Though these high resolution imaging techniques help one visualize these nano-structures, it is essential to understand the chemical nature of these materials and their growth mechanisms. Surface modifications can alter the bulk properties of these nanostructures and conventional characterization techniques associated with imaging systems are not capable of detecting these changes. Modern state of the art Scanning Auger Microscopy systems provide valuable elemental and chemical characterization for these structures with a spatial resolution better than $10 \mathrm{~nm}$ and depth resolutions of a few $\mathrm{nm}$. In this presentation we will review some unique features of the Scanning Auger Microscopy technique and highlight their applications in characterizing elemental and chemical structures in the areas of nanotechnology, metallurgy and semiconductors in conjunction with traditional microscopy techniques such as FIB cross section sample preparation.

In a state-of-the-art Scanning Auger system a coaxial electron gun and analyzer geometry (figure 1a) provides unique sensitivity and unobstructed vision needed to fully characterize the micro and nanostructure that exists on most real world samples. Figures $1 \mathrm{~b} \& 1 \mathrm{c}$ highlights an SEM image and the corresponding Ni Auger map obtained from all sides of the particulates and between the particles with very high and uniform sensitivity. Figure 2 shows a high resolution Al map of an AlGaAs/GaAs lattice. The Al map was acquired over 8 hours to illustrate the long term, high sensitivity analytical capability of modern scanning Auger systems. This analysis clearly defines the $4 \mathrm{~nm}$ line.

Focused ion beams are routinely used in the semiconductor industry to prepare buried defects for analyses. The presence of extremely small defects during the processing of a multi-layer semiconductor device can render a device completely useless. Figures $3 \mathrm{a}$ and $3 \mathrm{~b}$ show voltage contrast secondary electron images of a defective semiconductor device. Using FIB the defect area is exposed and the Scanning Auger electron spectroscopy map clearly identifies the missing copper via and the processing level where the failure occurred (Figure 3c).

Vertically aligned carbon nanofibers and nanocones (figure 4a) grown by plasma enhanced chemical vapor deposition have potential applications as field emission sources. STEM/EELS analyses of these nanocones are only able to identify the bulk elemental composition of these structures. High resolution Auger electron spectroscopy reveals a non-uniform surface chemical composition between several different nanocones. The quantitative elemental analysis (Figure 4b) as well as the imaging of different elemental (Figures 4c \& 4d) and chemical states, without imaging artifacts induced by the shapes, help elucidate the growth mechanisms of these nanostructures. 


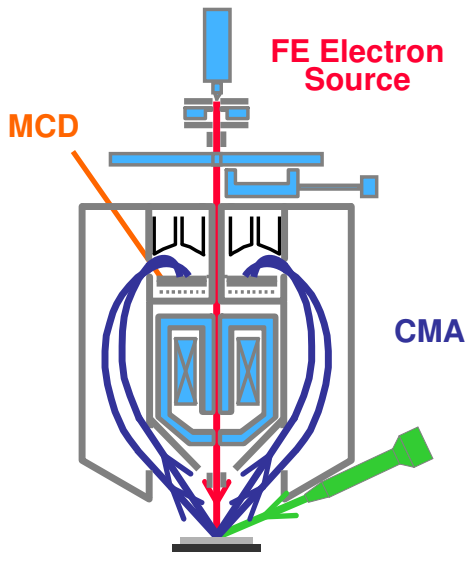

Figure 1a: Schematic or a CMA
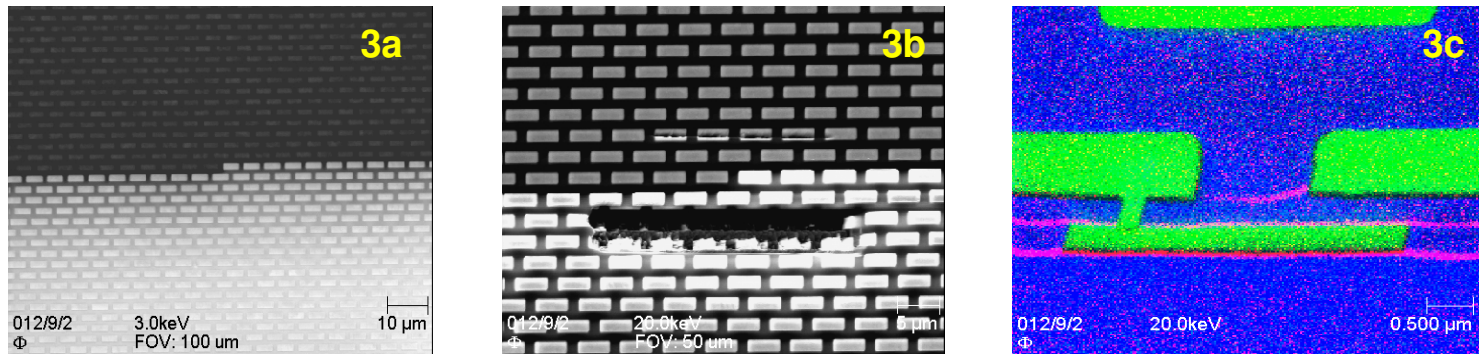

Figure 3: Voltage contrast image (a) of a defect exposed with FIB (b) and RGB elemental map (c) to identify the defect region. Red - Nitrogen, Green - Copper, Blue - Silicon
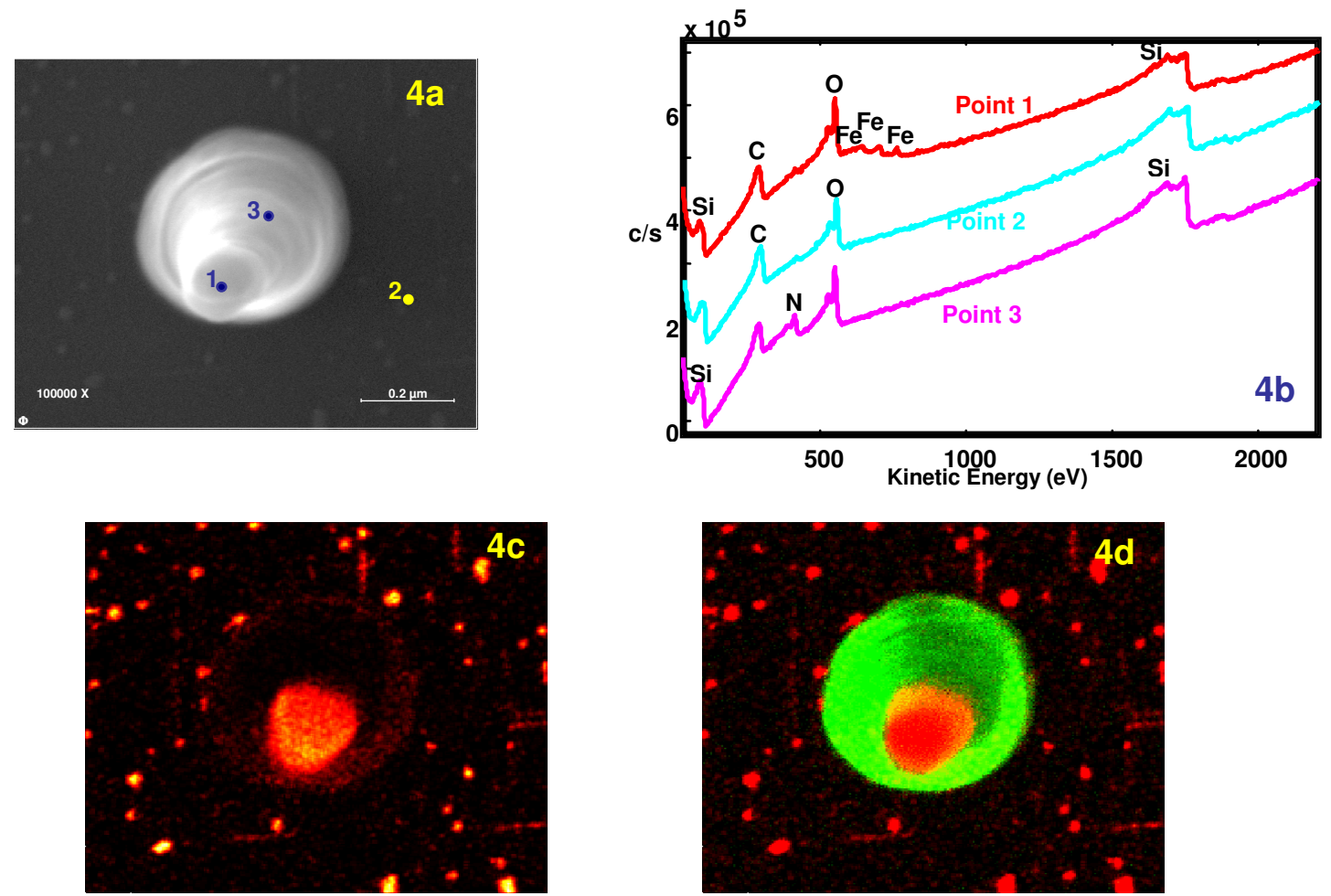

Figure 4: A typical top down SEM image of a nanocone (4a) and the small spot Auger analysis (4b) of the three selected areas. High resolution Auger map of the Fe seed (4c) on top of the nanocone and a composite image (4d) of Fe (red) and $\mathbf{N}$ (green) highlighting the advantage of the coaxial geometry of the analyzer 